\title{
Giant, Voltage-Actuated Deformation of a Dielectric Elastomer under Dead Load
}

\section{Citation}

Huang, Jiangshui, Tiefeng Li, Choon Chiang Foo, Jian Zhu, David R. Clarke, and Zhigang Suo. 2012. Giant, voltage-actuated deformation of a dielectric elastomer under dead load. Applied Physics Letters 100(4): 041911.

\section{Published Version}

doi:10.1063/1.3680591

\section{Permanent link}

http://nrs.harvard.edu/urn-3:HUL.InstRepos:9887630

\section{Terms of Use}

This article was downloaded from Harvard University's DASH repository, and is made available under the terms and conditions applicable to Open Access Policy Articles, as set forth at http:// nrs.harvard.edu/urn-3:HUL.InstRepos:dash.current.terms-of-use\#OAP

\section{Share Your Story}

The Harvard community has made this article openly available.

Please share how this access benefits you. Submit a story.

\section{Accessibility}




\title{
Giant, voltage-actuated deformation of a dielectric elastomer under dead load
}

Jiangshui Huang1, Tiefeng Li ${ }^{1,2}$, Choon Chiang $\mathrm{FoO}^{1,3}$, Jian $\mathrm{Zhu}^{1}$,

David R. Clarke ${ }^{1, \mathrm{a}}$, Zhigang Suo ${ }^{1, \mathrm{~b}}$

${ }^{1}$ School of Engineering and Applied Sciences, Harvard University, Cambridge, MA 02138

${ }^{2}$ Institute of Applied Mechanics, Zhejiang University, 38 Zheda Road, Hangzhou, Zhejiang 310027, China

3 Institute of High Performance Computing, 1 Fusionopolis Way, \#16-16 Connexis, Singapore 138632, Singapore

\begin{abstract}
Far greater voltage-actuated deformation is achievable for a dielectric elastomer under equal-biaxial dead load than under rigid constraint usually employed. Areal strains of $488 \%$ are demonstrated. The dead load suppresses electric breakdown, enabling the elastomer to survive the snap-through electromechanical instability. The breakdown voltage is found to increase with the voltage ramp rate. A nonlinear model for viscoelastic dielectric elastomers is developed and shown to be consistent with the experimental observations.
\end{abstract}

Keywords: Dielectric actuators, electromechanical instability, electric breakdown
a) clarke@seas.harvard.edu
b) suo@seas.harvard.edu 
When a voltage is applied across the thickness of a dielectric elastomer membrane, it reduces in thickness and increases in area. About a decade ago it was discovered that voltage-actuated strains over $100 \%$ could be achieved. ${ }^{1}$ The discovery has stimulated intense development of muscle-like transducers for broad range of applications, including soft robots, ${ }^{2}$ artificial limbs, ${ }^{3}$ Braille displays, ${ }^{4}$ microelectromechanical systems, ${ }^{5}$ bio-stimulation pads, ${ }^{6}$ adaptive optics, ${ }^{7-9}$ and generators. ${ }^{10-12}$

While elastomers can readily be stretched several times their initial length by a mechanical force, achieving large deformations by applying a voltage has been difficult. This difficulty can be appreciated as follows. Subject to voltage, a hard dielectric suffers electric breakdown at small deformation. For a voltage to induce large deformations, the dielectric must be soft. A soft dielectric, however, suffers electromechanical instability. As the thickness decreases in response to an applied voltage, the electric field increases. This leads to a positive feedback between the reduction in thickness and the increase in electric field, leading to electric breakdown. Theory predicts that, for elastomers under no mechanical load, the electromechanical instability occurs at an expansion of area about 70\%.13,14

It was therefore especially intriguing when an acrylic elastomer was demonstrated to attain voltage-induced strain over $100 \%{ }^{1} \quad$ The large actuation was achieved by pre-stretching a sheet, fixing it to a circular rigid frame, and then applying a voltage through the thickness of a small circular region in the center. The reported maximum voltage-actuated expansion in area was $158 \%$. Because the sheet was constrained by the frame, the mechanical force exerted on the active region reduced as the region expanded. 
This is so even when the active region is small. In this paper, we demonstrate that larger voltage-actuated strains can be produced when the elastomer is under equal-biaxial dead load, and that the attainable strains depend on the rate at which the voltage is applied. The observations are compared with a viscoelastic model of elastomer dielectrics, extending recent theory. ${ }^{15,16}$

In common with many previous studies of dielectric elastomers, we used thin sheets of VHB 4905, an acrylic elastomer produced by 3M. Circular discs, radius $R=17.5 \mathrm{~mm}$, were laser cut from the sheets, thickness $H=0.5 \mathrm{~mm}$. As illustrated in Fig. 1, constant radial forces $P$ was applied with weights attached, though a thread and pulley arrangement, to the edge of the disc. As the VHB elastomer is viscoelastic, the discs were first held under the fixed load for 30 minutes before applying any voltage. This time was found to be sufficient for the viscoelastic deformation to cease. The radius after this time under the dead load is denoted $r_{p r e}$, and the thickness $h_{p r e}$.

A thin layer of carbon grease (MG mechanicals, CAT. NO. 8462), of uniform thickness about $0.028 \mathrm{~mm}$, was then brushed on both the top and bottom surfaces of the discs to serve as compliant electrodes. Voltage was applied using a programmable voltage source (Keithley, model 230) and a high-voltage amplifier (Trek, model 610E). As the voltage was increased linearly in time, the expansion of the disc was recorded with a camera. From the recordings, the radius $r$ of the disc and the radial stretch, defined as $\lambda=r / R$, were obtained as a function of voltage. 
In the results presented in Fig 2, we used four values of fixed load, $P=20.0,25.5$, 31.0, and $36.5 \mathrm{~g}$, and three voltage ramp rates, $0.5 \mathrm{~V} / \mathrm{s}, 5 \mathrm{~V} / \mathrm{s}$, and $500 \mathrm{~V} / \mathrm{s}$. At all voltage rates, electric breakdown occurs at a low actuation strain under the smallest fixed load $(P=$ $20.0 \mathrm{~g}$ ), but when $P=25.5,31.0$ or $36.5 \mathrm{~g}$, the radius of the dielectric elastomer increases slowly at first and then abruptly, achieving large voltage-induced strains before electric breakdown. The abrupt increase is indicative of a viscoelastic snap-through behavior. While a small dead load does not eliminate the electromechanical instability, larger dead loads bring the mechanical pre-stretch closer to the physical stretching limit of the dielectric elastomers. Under dead load $P=25.5 \mathrm{~g}$ and at voltage ramp rate $500 \mathrm{~V} / \mathrm{s}$, the largest voltage-actuated strain, 488\% expansion in area, was achieved.

A strong dependence on the voltage ramp rate was observed over the range $0.5 \mathrm{~V} / \mathrm{s}$ to $500 \mathrm{~V} / \mathrm{s}$ (Fig. 2). The higher the voltage ramp rate, the larger the voltage required to actuate the elastomer deformation and the higher the voltage before breakdown occurs. The former result indicates that the elastomer becomes stiffer and the latter indicates that the dielectric strength becomes larger. The origin of the larger dielectric breakdown strength is not clear. It could be related to the observed increase in the stiffness or, alternatively, be due to a field-assisted, time dependent breakdown phenomenon associated charge migration through the elastomer.

To analyze the experimental observations, we develop a model for viscoelastic dielectric elastomers. When the radius of the disc increases from $R$ to $r$, its thickness reduces from $H$ to $h$. The elastomer is taken to be incompressible, so that $h r^{2}=H R^{2}$ and since 
$\lambda=r / R$ we can write $h=H \lambda^{-2}$. The stress due to the dead load is $\sigma=\lambda N P /(2 \pi H R)$, where $N$ is the number of individual weights. The electric field is $E=\lambda^{2} \Phi / H$. We adopt a nonlinear rheological model consisting of two parallel elements: a spring, $\alpha$, in parallel with another spring , $\beta$, and a dashpot with Newtonian fluid characteristics (Fig. 3a). ${ }^{17-19}$ For the spring $\alpha$, and the parallel element of spring and dashpot, the deformation is characterized by the stretch $\lambda$. The stretch in the spring $\beta$ is different, $\lambda^{e}$, and in the dashpot is $\xi$, so that $\lambda=\lambda^{e} \xi$. Dielectric elastomers show pronounced strain-stiffening as the stretch approaches the maximum elongation of its polymer chains. To account for strain-stiffening, we represent both springs by using the Gent model, ${ }^{20}$ and write

$$
\left(\frac{\lambda N P}{2 \pi H R}\right)+\varepsilon\left(\frac{\lambda^{2} \Phi}{H}\right)^{2}=\frac{\mu^{\alpha}\left(\lambda^{2}-\lambda^{-4}\right)}{1-\left(2 \lambda^{2}+\lambda^{-4}-3\right) / J_{\lim }^{\alpha}}+\frac{\mu^{\beta}\left(\lambda^{2} \xi^{-2}-\xi^{4} \lambda^{-4}\right)}{1-\left(2 \lambda^{2} \xi^{-2}+\xi^{4} \lambda^{-4}-3\right) / J_{\lim }^{\beta}}
$$

where $\varepsilon$ is the relative dielectric permittivity, $\mu^{\alpha}$ and $\mu^{\beta}$ are shear moduli, and $J_{\lim }^{\alpha}$ and $J_{\text {lim }}^{\beta}$ are constants related to the stiffening. The two terms on the left-hand side of (1) are the stresses due to the dead load and the Maxwell stress due to the voltage, respectively. The two terms on the right-hand side of (1) are the stresses carried by the two springs in the rheological model. In essence, the elasticity of the polymeric network is balanced by the sum of the applied stress and the Maxwell stress.

In the rheological model, the stress in the dashpot is the same as that in spring $\beta$ and is given as the last term in (1). The rate of deformation in the dashpot is $\xi^{-1} d \xi / d t$. To model the dashpot as a Newtonian fluid with viscosity $\eta$ we relate the rate of deformation to the stress on the dashpot: 


$$
\left.\frac{d \xi}{\xi d t}=\frac{\mu^{\beta}\left(\lambda^{2} \xi^{-2}-\xi^{4} \lambda^{-4}\right)}{6 \eta\left[1-\left(2 \lambda^{2} \xi^{-2}+\xi^{4} \lambda^{-4}-3\right) / J_{\lim }^{\beta}\right.}\right] .
$$

Once the mechanical and electrical loading functions, $P(t)$ and $\Phi(t)$, are prescribed, (1) and (2) evolve to give $\lambda(t)$ and $\xi(t)$.

To determine the purely mechanical deformation response of the VHB elastomer, we measured, at zero voltage, the stress-stretch response at two loading rates of $11.0 \mathrm{~g} / 5 \mathrm{~s}$ and 11.0 g/90 s. Fig. 3b compares the model, with fitting parameters shown in the inset, and the experimental data. The observed strain-stiffening is clearly captured by the model.

Using these parameters, and the reported permittivity $\varepsilon=3.98 \times 10^{-11} \mathrm{~F} / \mathrm{m}$, voltage-stretch curves were calculated (Fig. 4). The main features of the observations (Fig. 3) are reproduced. Also, consistent with experiment, the prediction shows that electromechanical instability occurs for small dead loads $(P=20.0 \mathrm{~g})$, but is eliminated for large dead loads. In our calculations, the instability occurs when the determinant of the Hessian of the free energy function vanishes. ${ }^{17}$ Furthermore, our model shows a stiffer response with increasing voltage rates, a trend in agreement of the experimental observation, but not previously predicted.

While the model predicts qualitatively the experimental observations, quantitative agreement has been difficult to achieve. The discrepancy is mainly due to the limitation of the simple viscoelastic model used here which invokes only one relaxation time. In reality, however, the elastomer appears to possess multiple relaxation times. In Fig. 3, the model is able to capture the short-time response of the elastomer, which is loaded from rest. However 
in Fig. 4, the elastomer is first pre-loaded for a long time, before it is actuated by voltage. Viscous relaxation occurs during this pre-loading period.

In summary, experiment demonstrates that a large dead load can eliminate electromechanical instability, allowing very large voltage-actuated strains, up to $488 \%$ expansion in area. Also, the elastomer appears to be stiffer at higher voltage ramp rates. These observations are qualitatively described by a nonlinear model of viscoelastic dielectrics. To achieve quantitative agreement, one should invoke a more realistic viscoelastic model. Specifically, the elastomer seems to possess multiple relaxation times, which may be incorporated in the model by introducing more parallel units of springs and dashpots. In our analyses, the membrane is subject to homogeneous and equal-biaxial deformation. For other geometries, deformation may be inhomogeneous, so that exact analysis requires solving boundary-value problems.

\section{Acknowledgements}

The work was supported by DARPA (W911NF-10-1-0113, Cephalopod-inspired Adaptive Photonic Systems). The authors would like to thank Dr. Samuel Shian for his assistance on Labview programming. CC Foo acknowledges A*STAR, Singapore for sponsoring his postdoctoral visit to Harvard University. 


\section{References}

1. R. Pelrine, R. Kornbluh, Q. Pei., J. Joseph, Science 287, 836 (2000).

2. G. Kovacs, L. During, S. Michel, G. Terrasi, Sensors Actuators A 155, 299 (2009).

3. E. Biddiss, and T. Chau, Med. Eng. \& Phys. 3o, 403 (2008).

4. Z.B. Yu, W. Yuan, P. Brochu, B. Chen, Z.T. Liu, Q.B. Pei, Appl. Phys. Lett. 95, 192904 (2009).

5. S. Rosset, M. Niklaus, P. Dubois, H.R. Shea, J. Microelectromechanical Sys. 18, 1300 (2009).

6. F. Carpi, G. Frediani, S. Tarantino, and D. De Rossi, Polymer Int. 59, 407 (2010).

7. C. Keplinger, M. Kaltenbrunner, N. Arnold, and S. Bauer, Proc. Nat. Acad. Sci. 107, 4505 (2010).

8. G. Kofod, D. N. McCarthy, J. Krissler, G. Lang, and G. Jordan, Appl. Phys. Lett. 94, 202901 (2009).

9. F. Carpi, G. Frediani, S. Turco, and D. De Rossi, Adv. Funct. Mater. 21, 4152 (2011).

10. T.G. McKay, B.M. O’Brien, E.P. Calius, I.A. Anderson, Appl. Phys. Lett. 98, 142903 (2011).

11. S.J.A. Koh, C. Keplinger, T.F. Li, S. Bauer, Z.G. Suo. IEEE/ASME Trans. Mechatronics 16, 33 (2011).

12. R. Kaltseis, C. Keplinger, R. Baumgartner, M. Kaltenbrunner, T.F. Li, P. Machler, R. Schwodiauer, Z.G. Suo, S. Bauer, Appl. Phys. Lett. 99, 162904 (2011).

13. K. H. Stark, C. G. Garton, Nature 176, 1225 (1955).

14. X.H. Zhao, Z.G. Suo, Appl. Phys. Lett. 91, 061921 (2007).

15. X.H. Zhao, Z.G. Suo, Phys. Rev. Lett. 104, 178302 (2010).

16. S.J.A. Koh, T.F. Li, J.X. Zhou, X.H. Zhao, W. Hong, J. Zhu, Z.G. Suo, J Polym. Sci. Polym. Phys. 49, 504 (2011).

17. X.H. Zhao, S.J.A. Koh, Z.G. Suo, Int. J. Appl. Mech. 3, 1 (2011).

18. W. Hong, J. Mech. Phys. Solids. 59, 637 (2011).

19. C. C. Foo, S. Q. Cai, S. J. A. Koh; S. Bauer, Z.G. Suo. Submitted for publication.

20. A. N. Gent, Rubber Chem. Tech. 69, 59 (1996). 


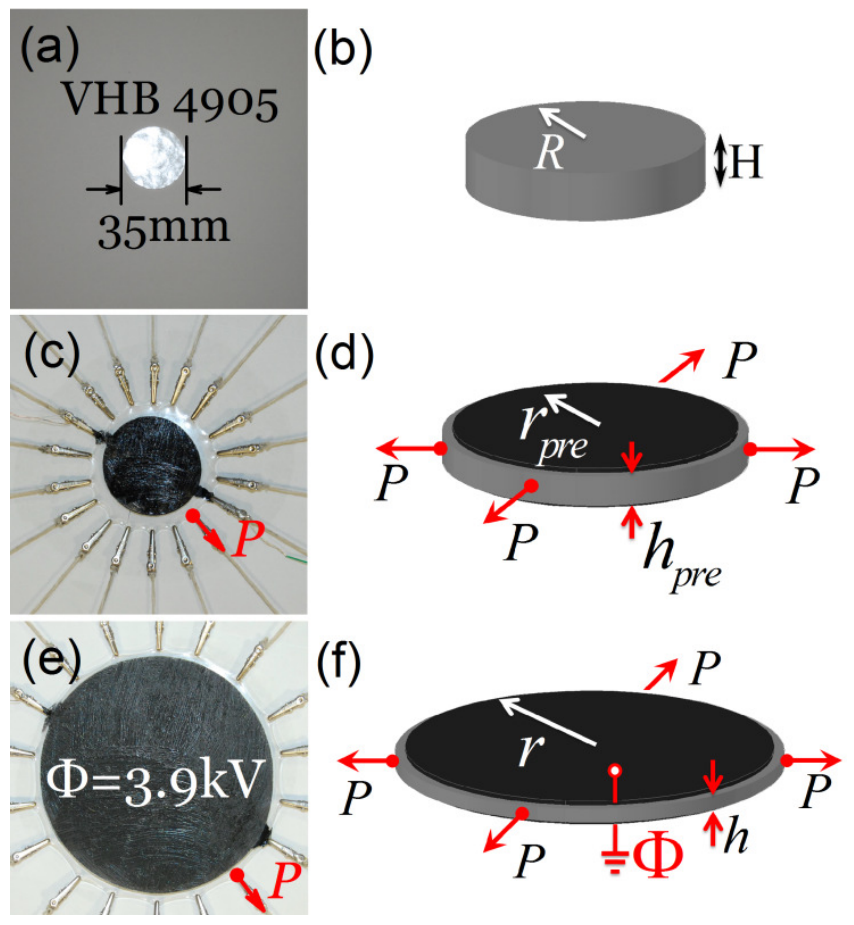

FIG. 1 (Color online) (a, b) Photograph and schematic of a circular disc of VHB, thickness $H$ $=0.5 \mathrm{~mm}$ and radius $R=17.5 \mathrm{~mm}$, before applying a voltage. (c) Attachment of loading weights (eighteen in number). (d) Under the dead load, the radius and thickness become $r_{\text {pre }}$ and $h_{\text {pre }}$, respectively. Both surfaces of the disc are coated with electrodes of carbon grease. (e, f) When both the dead load and voltage, $\Phi$, are applied, the radius $r$ increases and the thickness $h$ decreases. The photos $(\mathrm{a}, \mathrm{c}, \mathrm{e})$ are all taken at the same magnification. Comparison of (c) and (e) shows a voltage-actuated stretch of $r / r_{p r e}=2.4$ in this particular example. 

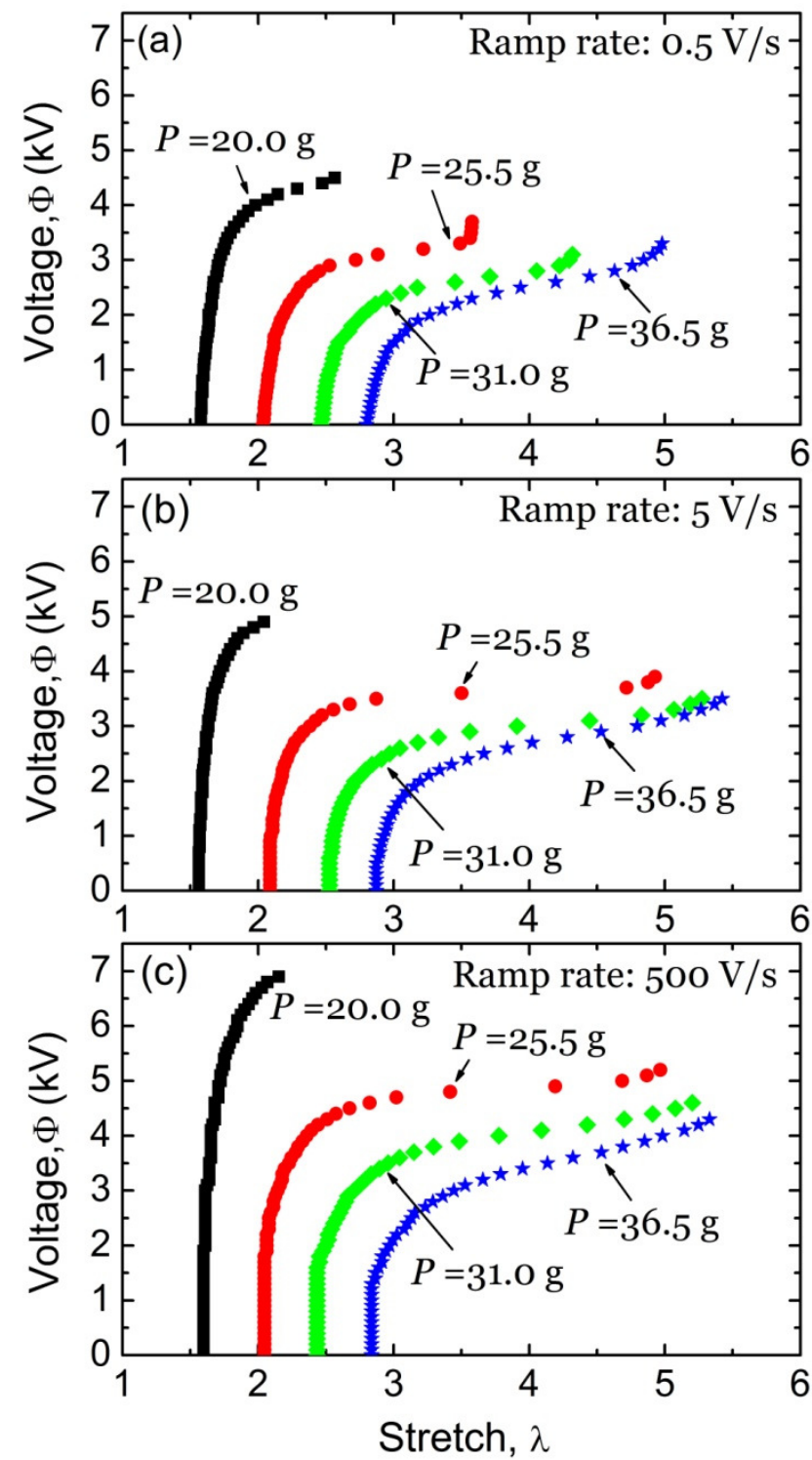

FIG. 2. (Color online) Under dead loads, the voltage-stretch curves are measured as the voltage is increased at rates of (a) $0.5 \mathrm{~V} / \mathrm{s}$, (b) $5 \mathrm{~V} / \mathrm{s}$, and (c) $500 \mathrm{~V} / \mathrm{s}$. The successive data points are plotted for equal time intervals of of $200 \mathrm{~s}, 20 \mathrm{~s}$, and $0.2 \mathrm{~s}$, for the three ramp rates, respectively. 
(a)
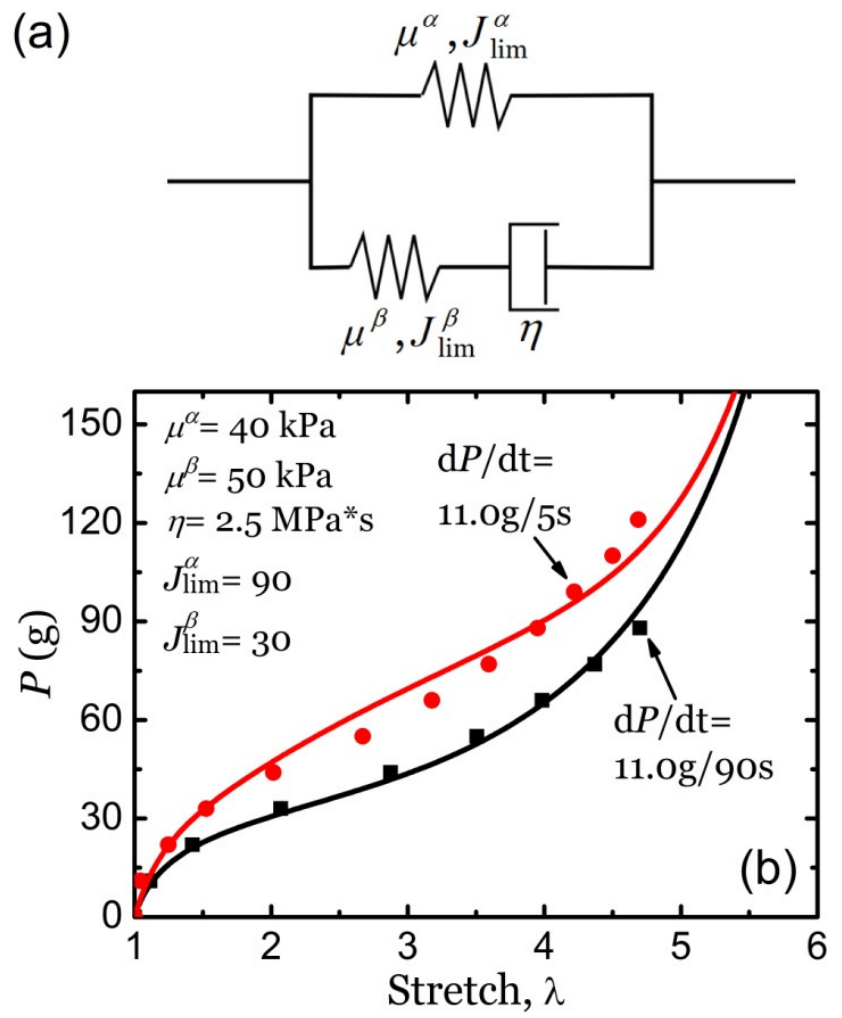

FIG. 3. (Color online) (a) Viscoelastic relaxation is modeled with a dashpot and two nonlinear springs. (b) With no voltage applied, the mechanical load vs stretch of the discs were measured at 2 loading rates of $11.0 \mathrm{~g} / 5 \mathrm{~s}$ and $11.0 \mathrm{~g} / 90 \mathrm{~s}$. The symbols are experimental data, while the curves show the fitting with the viscoelastic model. 

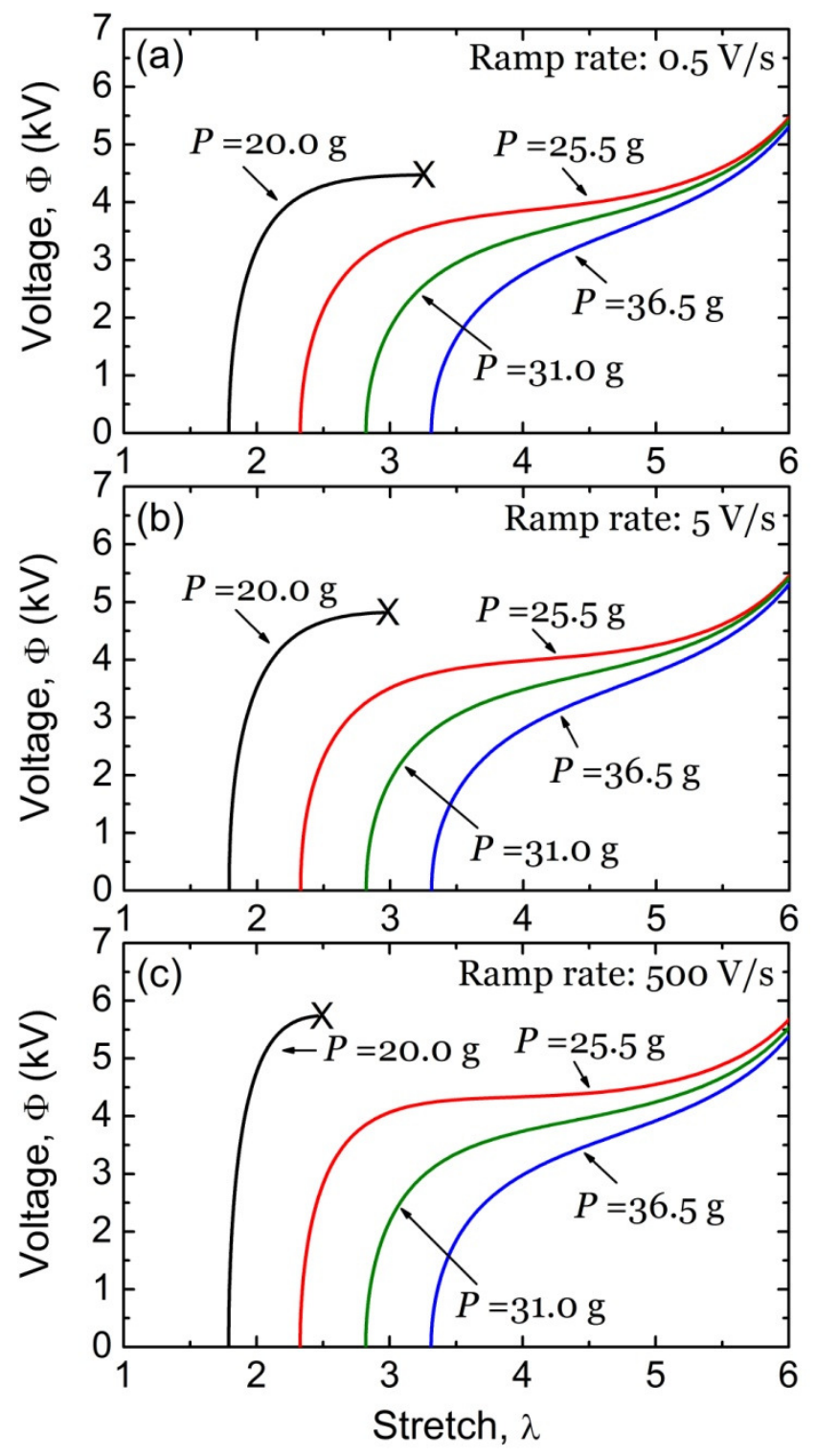

FIG. 4. (Color online) Under dead loads, dielectric elastomer discs were subjected to voltage at three different voltage ramp rates. The predicted voltage-stretch curves for various loads are shown. At low a dead load, the disk fails by electromechanical instability (denoted by the cross ' $\mathrm{X}$ '). 